\title{
Accelerated expansion of the Crab Nebula and evaluation of its neutron-star parameters
}

\author{
M. Bejger and P. Haensel
}

\author{
N. Copernicus Astronomical Center, Polish Academy of Sciences, Bartycka 18, 00-716 Warszawa, Poland \\ e-mail: bejger@camk.edu.pl; haensel@camk.edu.pl
}

Received 30 January 2003 / Accepted 16 April 2003

\begin{abstract}
A model of an accelerated expansion of the Crab Nebula powered by the spinning-down Crab pulsar is proposed, in which time dependence of the acceleration is connected to the evolution of pulsar luminosity. Using recent observational data, we derive estimates of the Crab neutron-star moment of inertia. Correlations between the neutron star moment of inertia and its mass and radius allow for rough estimates of the Crab neutron-star radius and mass. In contrast to the previously used constant-acceleration approximation, even for the expanding nebula mass of $\sim 7 M_{\odot}$ the results obtained within our model are not in conflict with the modern stiff equations of state of dense matter.
\end{abstract}

Key words. stars: neutron - stars: pulsars: individual: Crab - ISM: supernova remnants

\section{Introduction}

The AD 1054 supernova remnant, Crab Nebula, is probably the most often-observed object in the sky. Optical observations of its filaments made in the past century are sufficient to indicate that the motion of filaments is accelerated, $\dot{v}>0$. This accelerated expansion, connected to the local interstellar medium sweeping as well as the nebula radiation, is all powered by the Crab pulsar which was discovered in the center of the nebula in 1968 . The energy reservoir is constituted by the pulsar rotational energy, which loses energy at a rate $\dot{E}_{\text {rot }}=I \Omega \dot{\Omega}$, where $I$ is the pulsar moment of inertia and $\Omega$ and $\dot{\Omega}$ are angular frequency and its time derivative, both obtained from the pulsar timing. Assuming the balance between $\dot{E}_{\text {rot }}$ and the power of the nebula radiation and accelerated expansion in the interstellar medium, one gets a constraint on $I$, which in turn may be used to put a condition on the largely unknown equation of state (EOS) of dense matter.

Classical analysis along these lines was proposed and carried out by Manchester \& Taylor (1977). Some thirteen years later, it was carried out by one of us using more recent data on the Crab Nebula (Haensel 1990). In both cases, it has been assumed that $\dot{v}=$ const. during nebula expansion. Constraints derived by Manchester \& Taylor (1977) were weak and did not eliminate any of EOSs. The later analysis in Haensel (1990) pointed out the crucial dependence on the mass of the expanding nebula $M_{\text {neb }}$. The highest of the estimates of $M_{\text {neb }}$ available in the 1980s ruled out the softest EOSs.

The most recent estimates of the mass contained in the optical filaments are significantly higher than the previous ones $\left(4.6 \pm 1.8 M_{\odot}\right.$, Fesen et al. 1997). As we have recently shown, putting $M_{\text {neb }}=4.6 M_{\odot}$ in the classical $\dot{v}=$ const. expansion

Send offprint requests to: M. Bejger, e-mail: bejger@camk . edu.pl model eliminates nearly all existing EOSs except the stiffest ones (Bejger \& Haensel 2002). Actually, the situation can be even worse: the elementary model of type II supernovae predicts that a neutron star is a byproduct of explosion of an evolved star with mass $\gtrsim 8 M_{\odot}$. Matter seen as filaments constitutes only a part of the ejected mass, and with $M_{\text {neb }} \sim 7 M_{\odot}$ no realistic EOS can provide the Crab pulsar with sufficiently high $I$ to account for the $\dot{E}_{\text {rot }}$ needed. This would eliminate all existing realistic EOSs of dense matter.

Here we present a model of the Crab Nebula expansion which avoids the artificial approximation $\dot{v}=$ const. and is consistent with stiff EOSs even for $M_{\text {neb }} \sim 7 M_{\odot}$. We use $\dot{v}$ averaged in time, using a standard model of the pulsar frequency evolution. This assumption, based on elementary pulsar astrophysics, removes most of the drastic problems connected to high $M_{\text {neb }}$.

In Sect. 2 we summarize the observations and apply them to the description of the kinematics and energy budget of the Crab Nebula. In Sect. 3.1 we briefly summarize results obtained using the $\dot{v}=$ const. approximation. Our model for the accelerated expansion is presented in Sect. 3.2. It is used to evaluate $I$ of the Crab pulsar, which is then applied in Sect. 4 to derive constraints on the dense matter EOS. Finally, we apply recently derived formulae expressing $I$ in terms of the stellar mass and radius (Bejger \& Haensel 2002) to get constraints in the massradius plane for the neutron star and strange star model of the Crab pulsar.

\section{Observational facts and energy balance of the pulsar-nebula system}

The presently measured pulse period and the period derivative of the Crab pulsar are $P_{\mathrm{p}}=0.0334033 \mathrm{~s}$ and 
$\dot{P}_{\mathrm{p}}=4.20996 \times 10^{-13} \mathrm{~s} \mathrm{~s}^{-1}$ (Taylor et al. 1993), which corresponds to the angular frequency $\Omega_{\mathrm{p}}=188.101 \mathrm{~s}^{-1}$ and $\dot{\Omega}_{\mathrm{p}}=-2.37071 \times 10^{-9} \mathrm{~s}^{-2}$. The rotational energy of the neutron star is dissipated via the emission of particles, electromagnetic waves and through the interaction of the pulsar with the surrounding gas. The value of $\dot{\Omega}$ can be related to $\Omega$ by

$\dot{\Omega}=-K \Omega^{n}$,

where $K$ and $n$ are constants to be determined from the pulsar timing. In the c.g.s. units $K=4.66 \times 10^{-15}$. The breaking index $n$ can be expressed in terms of the measurable timing parameters $\Omega, \dot{\Omega}$, and $\ddot{\Omega}$, namely $n=\Omega \ddot{\Omega} / \dot{\Omega}^{2}$. Its value for the Crab pulsar, calculated using the 1982-1987 timing data, is $n=2.509 \pm 0.001$ (Lyne et al. 1998). We make a standard assumption that $n$ depends only on the pulsar magnetic field, whose configuration was fixed after formation of the pulsar (e.g., in less than a few months). In what follows we will count the pulsar age from that moment. Integration of the Eq. (1) from $t=0$ to $t=T=938 \mathrm{yr}$ (the reason for choosing this value of $T$ will become clear later) will give us the initial angular frequency $\Omega_{\mathrm{i}}$ and initial period $P_{\mathrm{i}}$ :

$\Omega_{\mathrm{i}}=\left[\Omega_{\mathrm{p}}^{1-n}-K T(n-1)\right]^{1 /(1-n)}=325.757 \mathrm{~s}^{-1}$,

$P_{\mathrm{i}}=\frac{2 \pi}{\Omega_{\mathrm{i}}}=0.0192880 \mathrm{~s}$

The loss of the rotational energy can be written as

$\dot{E}_{\mathrm{rot}}=\frac{\mathrm{d}}{\mathrm{dt}}\left(\frac{1}{2} I \Omega^{2}\right)=-I \Omega|\dot{\Omega}|$,

where small contribution resulting from the dependence of $I$ on $\Omega$ (the increase in $I$ is quadratic in $P_{\mathrm{ms}} / P_{\mathrm{p}}$, where the massshedding period $P_{\mathrm{ms}} \sim 1 \mathrm{~ms}$ ) was neglected. The rotational energy of the pulsar is transformed into radiation luminosity $\dot{E}_{\text {rad }}$ and the energy needed to support accelerated nebula expansion in the surrounding interstellar medium is $\dot{E}_{\text {exp }}$.

In order to make further calculations feasible, we will introduce an approximation of spherical symmetry. In principle, deviations from spherical symmetry can be accounted for by introducing corrections via "anisotropy factors" in the final results. For the time being, we have no sufficient observational information to implement such a procedure, and we will restrict ourselves to the spherically-symmetric model. Following Petersen (1998) we write the total radiated energy per unit time as

$\dot{E}_{\mathrm{rad}}(D) \simeq 1.25 \cdot\left(\frac{D}{D_{\mathrm{DF}}}\right)^{2} \times 10^{38} \mathrm{erg} \mathrm{s}^{-1}$,

where $D$ is the distance to the nebula in kpc. The value $D_{\mathrm{DF}}=$ $1.83 \mathrm{kpc}$ comes from the paper of Davidson \& Fesen (1985). When calculating $\dot{E}_{\text {exp }}$, we should take into account the fact that the nebula expands in the interstellar medium. We will approximate the nebula by an expanding spherical shell of radius $R_{\text {neb }}$. The shell expansion velocity is then $v=\dot{R}_{\text {neb }}$. The expanding shell will increase its mass by sweeping the interstellar medium

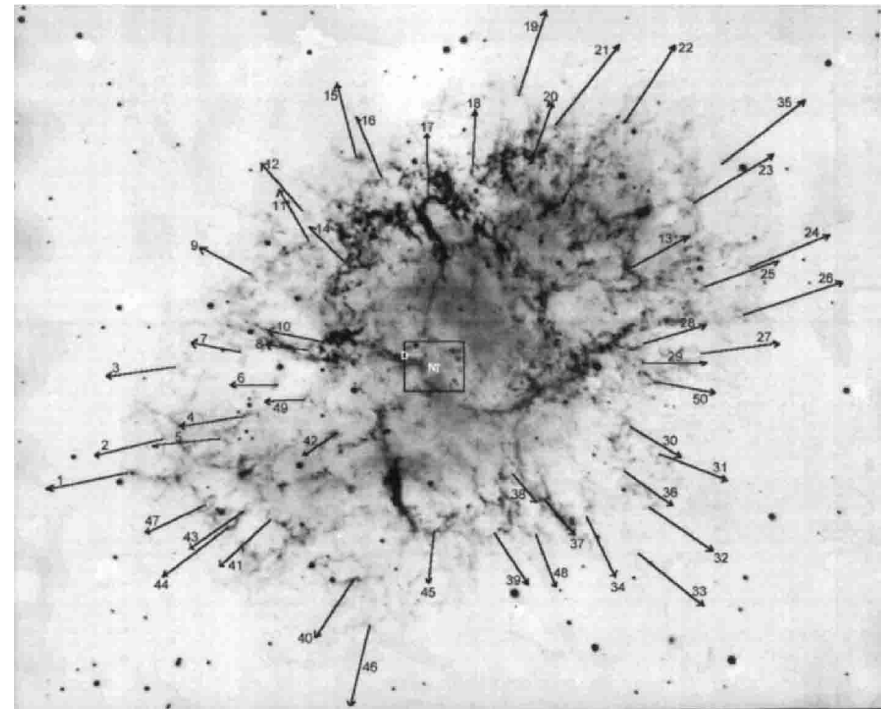

Fig. 1. Expansion of the Crab Nebula. Arrows represent motions of 50 optical filaments in the next $250 \mathrm{yr}$ at current expansion rates. From Nugent (1998), with kind permission of the author.

after accelerating it to its own velocity $v$. Therefore, the expression for $\dot{E}_{\text {exp }}$ reads

$\dot{E}_{\exp }=\frac{\mathrm{d}}{\mathrm{d} t}\left(\frac{1}{2} M_{\mathrm{neb}} v^{2}\right)=M_{\mathrm{neb}} v \dot{v}+\frac{1}{2} \dot{M}_{\mathrm{neb}} v^{2}$,

where $M_{\text {neb }}$ is the mass of the nebula. Mass of the interstellar hydrogen added to the nebula per unit time during the expansion of nebula in the interstellar medium is

$\dot{M}_{\mathrm{neb}}=4 \pi R_{\mathrm{neb}}^{2} n_{\mathrm{H}} m_{\mathrm{H}} v$,

where $m_{\mathrm{H}}$ denotes the hydrogen atom mass and $n_{\mathrm{H}}$ is the number density of hydrogen atoms in space around the nebula. For our computation we use the value $n_{\mathrm{H}}=0.2 \mathrm{~cm}^{-3}$ estimated by Manchester \& Taylor (1977).

Our spherical-shell model is the simplest possible representation of Crab Nebula, which is famous for its rather complicated crab-like shape. The value of $R_{\text {neb }}$ will be evaluated as the mean for an ellipsoid which is a more precise model of the shape of the Crab Nebula. Assuming $D=D_{\mathrm{DF}}$, one gets then $R_{\text {neb }}=1.25$ pc (see e.g. Douvion et al. 2001).

The present mass of the Crab Nebula, $M_{\text {neb }}$, will play a central role in our model. Its observational estimation is very difficult - in the last two decades the estimated value has varied from 2-3 $M_{\odot}$ (Davidson \& Fesen 1985), through 1-2 $M_{\odot}$ (MacAlpine \& Uomoto 1991) to $4.6 \pm 1.8 M_{\odot}$ (Fesen et al. 1997).

The expanding nebula shell is filled with optically shining filaments, whose motion can be measured by comparing the filament positions on high-resolution photographs taken more than 15-20 years apart (Duncan 1939; Trimble 1968; Wyckoff \& Murray 1977; Nugent 1998). In the present paper we will use the most recent results obtained by Nugent (1998). By comparing positions of 50 identifiable bright filaments on highresolution plates taken in 1939, 1960, 1976, and 1992, Nugent 
calculated the mean velocity of their expansion. His results are visualized in Fig. 1, which was for us a source of inspiration for studying the Crab Nebula dynamics. By projecting the straight-line constant velocity motion of filaments backward in time, Nugent obtained convergence of filaments trajectories at AD $1130 \pm 16$ yr. His result was in accordance with previous estimate of Trimble (1968). Had the nebula expanded at a constant $v$, this would be the moment of Crab supernova explosion. However, the date recorded by Chinese astronomers is AD 1054 , which is $\Delta=76 \mathrm{yr}$ earlier. Therefore, the expansion had a non-zero acceleration $\dot{v}$. During expansion, $v$ increased from initial $v_{\mathrm{i}}$ to the present $v_{\mathrm{p}}$, known also from the spectral measurements (e.g. Sollerman et al. 2000), $v_{\mathrm{p}} \sim 1.5 \times 10^{8} \mathrm{~cm} \mathrm{~s}^{-1}$.

\section{Crab Nebula dynamics and bounds for the moment of inertia of its neutron star}

\subsection{Constant acceleration}

In view of the lack of information on the time dependence of $\dot{v}$ during the nebula lifetime, the most natural approximation is to consider it as constant in time. This is the approximation used in previous studies (Manchester \& Taylor 1977; Haensel 1990; Bejger \& Haensel 2002). This constant value of acceleration will be denoted by $\dot{v}_{\mathrm{c}}$, and can be calculated from the existing data using the formula

$\dot{v}_{\mathrm{c}}=\frac{2 \Delta v_{\mathrm{p}}}{T^{2}}$,

where $T=938 \mathrm{yr}$ is the lifetime of the nebula from birth in 1054 AD to Nugent's photographic evaluation in 1992. Putting numerical values, we get $\dot{v}_{\mathrm{c}}=0.82 \times 10^{-3} \mathrm{~cm} \mathrm{~s}^{-2}$.

The knowledge of the present $v$ and $\dot{v}$ allows one to get an expression for the Crab pulsar moment of inertia. This expression results from the condition that the loss of the kinetic rotational energy of the pulsar should be sufficient to support $\dot{E}_{\mathrm{rad}}+\dot{E}_{\mathrm{exp}}$,

$$
\begin{aligned}
I_{\mathrm{Crab}} \geq & {\left[\dot{E}_{\mathrm{rad}}(D)+M_{\mathrm{neb}} v \dot{v}\right] /(\Omega|\dot{\Omega}|) } \\
& +2 \pi R_{\mathrm{neb}}^{2} n_{\mathrm{H}} m_{\mathrm{H}} v^{3} /(\Omega|\dot{\Omega}|) .
\end{aligned}
$$

The above equation is generally valid and does not involve an assumption of time dependence of $v$ and $\dot{v}$.

From Eq. (9), using $M_{\text {neb }}=4.6 M_{\odot}, D=1.83 \mathrm{kpc}, v=v_{\mathrm{p}}=$ $1.5 \times 10^{8} \mathrm{~cm} \mathrm{~s}^{-1}, \dot{v}=\dot{v}_{\mathrm{c}}=0.82 \times 10^{-3} \mathrm{~cm} \mathrm{~s}^{-2}$ and $\Omega_{\mathrm{p}}\left|\dot{\Omega}_{\mathrm{p}}\right|=$ $4.459 \times 10^{-7} \mathrm{~s}^{-3}$ we get an estimate of a lower bound on $I_{\text {Crab }}$, labeled with "c" from the constant acceleration assumption,

$$
\begin{aligned}
I_{\mathrm{Crab}, 45} \geq I_{45}^{(\mathrm{c})}= & 0.28\left(\frac{D}{D_{\mathrm{DF}}}\right)^{2}+2.53 \frac{M_{\mathrm{neb}}}{4.6 M_{\odot}} \\
& +0.23\left(\frac{R_{\mathrm{neb}}}{1.25 \mathrm{pc}}\right)^{2} \frac{n_{\mathrm{H}}}{0.2 \mathrm{~cm}^{-3}}
\end{aligned}
$$

where $I_{45} \equiv I / 10^{45} \mathrm{~g} \mathrm{~cm}^{2}$. With our choice of parameters, this equation yields $I_{45}^{(\mathrm{c})}=3.04$. As shown in our previous paper (Bejger \& Haensel 2002), such a value of $I_{\text {Crab }}$ requires a very stiff EOS of dense matter. Therefore, the constraint on the maximum moment of inertia for a dense matter EOS, $I_{\max }$, which should satisfy the inequality $I_{\max }>I_{\text {Crab }}$, is very strong. Actually, the problem can become quite dramatic if the expanding shell contains a typical amount of mass ejected in a type II supernova, because for $M_{\text {neb }} \gtrsim 7 M_{\odot}$ all existing realistic EOSs are ruled out by the $I_{\max }>I_{\text {Crab }}$ condition. However, as we show in the next section this may just result from the unrealistic character of the assumption $\dot{v}=$ const.

\subsection{Time-dependent acceleration}

As we can see, the acceleration term is largely dominating on the r.h.s. of Eq. (10). We will assume that this dominance was valid also in the past, after some initial short-term period $(<3 \mathrm{yr})$ in which the Crab Nebula was powered by the sources connected to supernova itself (i.e., ${ }^{56} \mathrm{Ni}$ radioactive decay heating). Therefore, the loss of the pulsar rotational kinetic energy goes mainly into accelerating the nebula,

$I \Omega|\dot{\Omega}| \simeq M_{\mathrm{neb}} v \dot{v}$

As we argued before, at $P>P_{\mathrm{i}}=19 \mathrm{~ms}$ the dependence of $I$ on $P$ (and therefore on time) is negligible. On the other hand, the total increase of the nebula mass since 1054 due to the sweeping of the interstellar medium can be estimated as

$\Delta M_{\text {neb }}=\frac{4}{3} \pi R_{\text {neb }}^{3} n_{\mathrm{H}} m_{\mathrm{H}}=0.04 M_{\odot}$.

This increase can be neglected compared to the present nebula mass, so that the assumption $M_{\text {neb }} \simeq$ const. is valid. Using Eq. (11) we can therefore approximately express $v \dot{v}$ at any moment in the past as

$v \dot{v} \simeq \frac{I \Omega|\dot{\Omega}|}{M_{\text {neb }}}$

During 938 yr of expansion, $v$ increased by some $2 \Delta / T \sim 16 \%$, (assuming $\dot{v}=$ const., see previous subsection) which is a relatively small change compared to the change in $\Omega \mid \dot{\Omega}$. Namely, equation

$\Omega|\dot{\Omega}|=K \Omega^{n+1}=K \Omega^{3.509}$

implies that during nebula lifetime the present $\Omega|\dot{\Omega}|$ decreased by a factor

$\left(\frac{\Omega_{\mathrm{i}}}{\Omega_{\mathrm{p}}}\right)^{3.509}=\left(\frac{P_{\mathrm{p}}}{P_{\mathrm{i}}}\right)^{3.509}=6.84$.

This indicates a rather strong dependence of the product $v \dot{v}$ on time. However, in view of a rather small increase in $v$, the time dependence of $v \dot{v}$ results mainly from a strong decrease of $\dot{v}$,

$\dot{v}(t) \simeq \frac{I \Omega|\dot{\Omega}|}{M_{\mathrm{neb}} v_{\mathrm{av}}}$,

where we approximated $v$ by its time-averaged value $v_{\mathrm{av}}=$ $R_{\text {neb }} / T$. The average value of the acceleration can be calculated from

$\dot{v}_{\mathrm{av}}=\frac{1}{T} \int_{0}^{T} \dot{v} \mathrm{~d} t$. 
Using previously derived expressions, we get

$\dot{v}_{\mathrm{av}}=\frac{I}{M T_{\mathrm{neb}} v_{\mathrm{av}}} \int_{\Omega_{\mathrm{i}}}^{\Omega_{\mathrm{p}}} \Omega|\dot{\Omega}| \mathrm{d} t=\frac{I}{2 T M_{\mathrm{neb}} v_{\mathrm{av}}}\left(\Omega_{\mathrm{i}}^{2}-\Omega_{\mathrm{p}}^{2}\right)$.

On the other hand, in the constant-acceleration model one would get

$I \Omega_{\mathrm{p}}\left|\dot{\Omega}_{\mathrm{p}}\right| \simeq M_{\mathrm{neb}} v_{\mathrm{av}} \dot{\mathrm{c}}_{\mathrm{c}}$.

Therefore,

$\frac{\dot{v}_{\mathrm{av}}}{\dot{v}_{\mathrm{c}}} \simeq \frac{\Omega_{\mathrm{i}}^{2}-\Omega_{\mathrm{p}}^{2}}{2 T \Omega_{\mathrm{p}}\left|\dot{\Omega}_{\mathrm{p}}\right|}=2.67$.

The above result indicates that under conditions prevailing during the Crab Nebula expansion the assumption $\dot{v}=$ const. is not valid. In what follows, we will use the approximation $I \Omega \dot{\Omega} \simeq M_{\mathrm{neb}} v \dot{v}$.

Within our model we can determine the present value of acceleration of the nebula expansion, using the method described below. We start with an elementary formula

$v_{\mathrm{p}}=v_{\mathrm{i}}+\int_{0}^{T} \dot{v} \mathrm{~d} t$.

Another elementary relation determines the average speed of expansion,

$v_{\mathrm{av}}=\frac{1}{T} \int_{0}^{T} v \mathrm{~d} t=\frac{R_{\mathrm{neb}}}{T}=1.3 \times 10^{8} \mathrm{~cm} \mathrm{~s}^{-1}$.

Changing the variable $t$ into $\Omega$, and carrying out the integrations over $\Omega$, we finally get a system of two equations for $v_{\mathrm{i}}$ and for $I$. The explicit form of this system of equations is:

$$
\begin{aligned}
v_{\mathrm{p}}=v_{\mathrm{i}} & +\frac{I\left(\Omega_{\mathrm{i}}^{2}-\Omega_{\mathrm{p}}^{2}\right)}{2 M_{\mathrm{neb}} v_{\mathrm{av}}}, \\
R_{\mathrm{neb}}= & v_{\mathrm{i}} T+\frac{I \Omega_{\mathrm{i}}^{3-n}}{2(3-n)(1-n) K M_{\mathrm{neb}} v_{\mathrm{av}}} \\
& \times\left(2+\left(\Omega_{\mathrm{p}} / \Omega_{\mathrm{i}}\right)^{3-n}\left[1-n-(3-n)\left(\Omega_{\mathrm{i}} / \Omega_{\mathrm{p}}\right)^{2}\right]\right),
\end{aligned}
$$

where the Crab pulsar timing constants $n$ and $K$ are given in Sect. 2. As a result we get $v_{\mathrm{i}}=0.93 \times 10^{8} \mathrm{~cm} \mathrm{~s}^{-1}$, and for an assumed $M_{\text {neb }}$ we are thus able to calculate the value of $I_{\text {Crab }}$. For the central and the upper value of $M_{\text {neb }}$ obtained by Fesen et al. (1997) we get the following numbers:

$$
\begin{aligned}
& M_{\text {neb }}=4.6 M_{\odot} \quad \Longrightarrow \quad I_{\text {Crab }, 45}>1.93, \\
& M_{\text {neb }}=6.4 M_{\odot} \quad \Longrightarrow \quad I_{\text {Crab }, 45}>2.68 .
\end{aligned}
$$

The value of $v_{\mathrm{i}}$ deserves a comment. It indicates that the initial energy of expanding filaments-shell,

$$
E_{\mathrm{kin}, \mathrm{i}}^{\text {shell }}=4.6 \times 10^{49} \frac{M_{\mathrm{neb}}}{4.6 M_{\odot}}\left(\frac{v_{\mathrm{i}}}{10^{8} \mathrm{~cm} \mathrm{~s}^{-1}}\right)^{2} \mathrm{erg}
$$

is much smaller than the canonical value expected for the corecollapse supernovae, $10^{51} \mathrm{erg}$. The missing kinetic energy may reside in a fast-moving outer shell of the supernova remnant (Chevalier 1977). Some evidence for the existence of such a fast-moving outer shell was found using far-ultraviolet and optical HST observations (Sollerman et al. 2000).

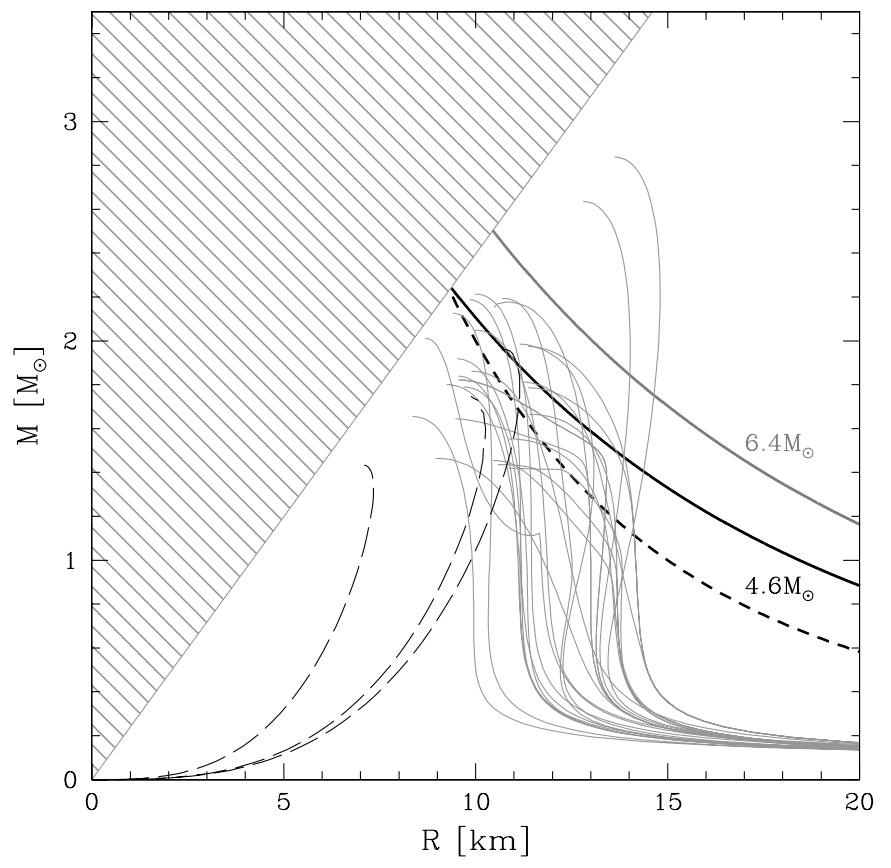

Fig. 2. The estimates for the moment of inertia of the Crab pulsar plotted on the radius-mass diagram, when $M_{\text {neb }}=4.6 M_{\odot}$, and $M_{\text {neb }}=6.4 M_{\odot}$. Thin lines represents various equations of state, dotted - neutron stars, long-dashed - strange stars. The set of thirty EOSs is the same as in Bejger \& Haensel (2002). In order to get constraints in $M-R$ plane, we use empirical $I(M, R)$ relations calculated by Bejger \& Haensel (2002) for neutron stars (thick solid lines) and strange stars (thick dashed line). The shaded area is excluded by General Relativity and the $v_{\text {sound }} \leq c$ condition.

\section{Constraints on the EOS, and M, and R of the Crab pulsar}

Within a simple astrophysical model of the time-dependent acceleration of the Crab Nebula expansion, we deduce constraints on dense matter EOS. These constraints depend on the mass of the Crab Nebula.

For a central value obtained by Fesen et al. (1997), in the constant-acceleration model one has $I_{\mathrm{Crab}, 45}>3.04$ which could be allowed only by the stiffest EOSs with $M_{\max }>2 M_{\odot}$ (Bejger \& Haensel 2002). With time-dependent acceleration we get $I_{\text {Crab }}$ which is some $40 \%$ lower, and this would exclude only soft EOSs and those EOSs which are strongly softened at supra-nuclear density (due to the presence of hyperons or a phase transition). Within our model of $\dot{v}(t)$ the Crab pulsar can also power the nebula with the uppermost value obtained by Fesen et al. (1997). We get then $I_{\text {Crab,45 }}\left(6.4 M_{\odot}\right)=2.68$, which leaves us with only a very stiff EOS with $M_{\max }>2 M_{\odot}$. Even $M \sim 7 M_{\odot}$ could be accommodated by existing stiff EOSs of matter composed of nucleons and leptons. Within constantacceleration models, such values of $M_{\text {neb }}$ rule out all existing realistic EOS of dense matter.

Using an empirical but actually very precise relation between the moment of inertia, mass of the star and the corresponding radius for neutron stars and strange quark stars 
(Bejger \& Haensel 2002), we plotted curves $I(M, R)=I_{\text {Crab }}$ in the mass-radius diagram (Fig. 2). From this plot we deduce constraints on the mass and radius of the Crab neutron star. If $M_{\text {neb }}=4.6 M_{\odot}$ then the neutron star has $M>1.5 M_{\odot}$ and $R=11-15 \mathrm{~km}$. If the Crab pulsar is a strange star (a rather unlikely situation because of glitches, see Alpar 1987), then it has to have mass $M>1.7 M_{\odot}$ and $R=10-11 \mathrm{~km}$. If $M_{\text {neb }}=6.4 M_{\odot}$, then the EOS should be stiff, and we get $M>1.7 M_{\odot}$ and $R=12-15 \mathrm{~km}$; moreover, the strange star model is ruled out.

Assuming that the progenitor star had mass $\gtrsim 8 M_{\odot}$ which roughly corresponds to a nebula mass $M_{\text {neb }} \sim 7 M_{\odot}$, the Crab neutron star should be even more massive and the lowest accepted $R$ is even larger. For such a mass of the nebula the massradius constraint definitely favors a stiff EOS. This constraint cannot be reconciled with high-density softening due to hyperonisation, or exotic phases of dense matter i.e. the strangequark matter. The derived Crab pulsar-neutron star mass limits are all larger than the 1.35-1.4 $M_{\odot}$ values measured with great precision in binary pulsar systems which may reflect a different formation scenario.

Acknowledgements. We would like to thank R. Nugent for the permission to use Fig. 1 in our paper. This work was partially supported by the KBN grants No. 5 P03D 02020 and 2 P03D 00422.

\section{References}

Alpar, M. A. 1987, Phys. Rev. Lett., 58, 2152

Bejger, M., \& Haensel, P. 2002, A\&A, 396, 917

Chevalier, R. A. 1977, in Supernovae, ed. D. N. Schramm (Dordrecht: Reidel), 53

Davidson, K., \& Fesen, R. A. 1985, ARA\&A, 23, 119

Douvion, T., Lagage, P. O., Cesarski, C. J., \& Dwek, E. 2001, A\&A, 373,281

Fesen, R. A., Shull, M. J., \& Hurdford, P. A. 1997, AJ, 113, 354

Duncan, J. 1939, ApJ, 89, 482

Haensel, P. 1990, in The magnetospheric Structure and Emission Mechanisms of Radio Pulsars, ed. T. H. Hankins, J. M. Rankin, \& J. Gil (Poland: Pedagogical University Press, Zielona Góra), IAU Colloq. 128, 217

Lyne, A. G., Pritchard, R. S., \& Smith, F. G. 1988, MNRAS, 233, 667

MacAlpine, G. M., \& Uomoto, A. 1991, AJ, 102, 218

Manchester, R. N., \& Taylor, J. H. 1977, Pulsars (San Francisco: W. H. Freeman and Company)

Nugent, R. 1998, PASP, 110, 831

Petersen, L. E. 1998, RevMexAA (Serie de Conferencias), 7, 81

Sollerman, J., Lundqvist, P., Lindler, D., et al. 2000, ApJ, 537, 861

Taylor, J. H., Manchester, R. N., \& Lyne A. G. 1993, ApJS, 88, 529 (on-line: http://pulsar. ucolick.org/catalog)

Trimble, V. L. 1968, AJ, 73, 535

Wycoff, S., \& Murray, C. A. 1977, MNRAS, 180, 717 